\title{
Incremental Latin Hypercube Sampling for Lifetime Stochastic Behavioral Modeling of Analog Circuits
}

\author{
*Yen-Lung Chen, ${ }^{\dagger}$ Wei Wu, ${ }^{*}$ Chien-Nan Jimmy Liu and ${ }^{\dagger}$ Lei He \\ *Electrical Engineering Dept., National Central University, Taiwan \\ ${ }^{\dagger}$ Electrical Engineering Dept., University of California, Los Angeles, CA, USA \\ Email: keith.ylchen@gmail.com,weiw@seas.ucla.edu,jimmy@ee.ncu.edu.tw, lhe@ee.ucla.edu
}

\begin{abstract}
In advanced technology node, not only process variations but also aging effects have critical impacts on circuit performance. Most of existing works consider process variations and aging effects separately while building the corresponding behavior models. Because of the time-varied circuit property, parametric yield need to be reanalyzed in each aging time step. This results in expensive simulation cost for reliability analysis due to the huge number of circuit simulation runs. In this paper, an incremental Latin hypercube sampling (LHS) approach is proposed to build the stochastic behavior models for analog/mixed-signal (AMS) circuits while simultaneously considering process variations and aging effects. By reusing previous sampling information, only a few new samples are incrementally updated to build an accurate stochastic model in different time steps, which significantly reduces the number of simulations for aging analysis. Experiments on an operational amplifier and a DAC circuit achieve $242 x$ speedup over traditional reliability analysis method with similar accuracies.
\end{abstract}

\section{INTRODUCTION}

With shrinking device size in deep submicron process, parametric yield loss due to process variations has become a critical issue, especially for analog/mixed signal (AMS) circuits. Those random variations on circuit parameters impose a lot of uncertainties on the circuit performance. Apart from the process variation, the transistor aging is another major reliability concern for analog circuits. The major aging effects include Negative-bias temperature instability (NBTI) [1], [2] and Hot-carrier injection (HCI) [1], [3]. Both of them can change the threshold voltage of a transistor over time and impact the circuit performance.

To understand the uncertainty introduced by process variations, statistical circuit analysis is required to analyze the circuit during the pre-silicon phase. It helps designers improve their design yield at an early stage and reduce re-design cycles and re-spin cost. As the golden standard of statistical circuit analysis, Monte Carlo (MC) method [4] draws massive samples according to the process variations and outputs the distribution of the circuit performance, which is also referred as circuit behavior in this paper.

With aging effects, it is challenging to calculate the lifetime of a circuit while considering process variation and performance degradation simultaneously. Even though lots of efforts have been spent to reduce the runtime of a single simulation [5], [6], [7], the total runtime can still be prohibitively long when $\mathrm{MC}$ simulation is required at each aging time step. There are several approaches to analyze the circuit performance at a specific aging time step without creating behavior model for lifetime yield analysis [8], [9]. They utilize a quadraticmodel to estimate the worst case distance (WCD) for a quick estimation of the design yield. However, the aging ratio varies at each time step and that induces the prediction error to this non-linear aging model.

In practice, there are several approaches to improve the MC simulation efficiency at each time step: such as efficient sampling methods[10], [11] and stochastic modeling[12], [13], [14], [15].

Unlike MC simulation that uses massive random samples [16], several existing sampling methods have been proposed to achieve acceptable coverage with fewer samples [10], [11]. Instead of using random samples, Quasi-Monte Carlo (QMC) method uses a low-discrepancy sequence (a.k.a. quasi-random sequence), which has a faster rate of convergence [10]. Another alternative to obtain the pseudo-random sequence is the Latin Hypercube Sampling (LHS) method [11]. It guarantees all the samples with low dependence to obtain faster coverage rate in most cases.

On the other hand, the stochastic modeling is proposed to obtain an approximated circuit behavior with reduced number of samples [12], [13], [14], [15]. For example, [12] fits the circuit behavior to a number of normally distributed variables using linear regression, which may become inaccurate for strongly nonlinear circuits. PEM [13] estimates the unknown distribution of circuit behavior with stochastic orthogonal polynomials. However, it requires prior knowledge of the distribution type that is unavailable in practice.

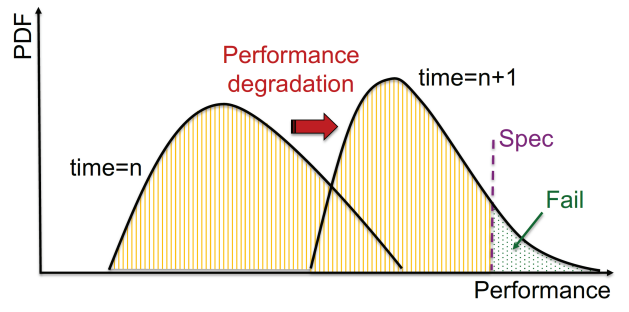

Fig. 1. The performance degradation due to aging effects

Even though the sampling methods and stochastic modeling relief the simulation burden in MC analysis, they do not provide an efficient solution for statistical circuit analysis considering process variation and aging simultaneously. Repeated simulations are still required at each time step due to the transistor aging over time. In fact, circuit aging is a gradual process, which does not change the transistor behavior 
drastically in each time step. Hence, there could be a large portion of overlap between the sample space in a time step and the one after, as illustrated in Fig. 1. Tailoring an efficient sampling approach to take advantage of the overlap is the major motivation behind this work.

In this paper, we propose an incremental LHS sampling method for simultaneous variability and aging analysis. Instead of independently simulating new samples at each time step, we analyze the samples drew in the previous time step, and incrementally add extra samples and remove the redundant ones from the sample space. In the experiments on an operational amplifier (OPA) circuit, incremental sampling reduces more than $150 \%$ samples for all the time steps on average. Most importantly, the proposed incremental sampling still guarantees the property of LHS at each time step, i.e. the samples are exactly distributed according to their distribution in each sample space.

Furthermore, stochastic modeling is also applied in the proposed approach to further reduce the required samples at each time step. In this work, PEM [13] is adopted to approximate the distribution to a closed-form function. There are a variety of choices of the stochastic modeling method, including APEX [14], MAXENT [15]. It can be easily replaced with other methods since the stochastic modeling step is independent to the incremental sampling step.

The remainder of this paper is organized as follows. Section II introduces the aging effects, moment matching and LHS techniques. The key ideas of the proposed approach for Incremental Latin Hypercube Sampling are discussed in Section III. Section IV demonstrates the experimental results and shows the improvements compared to the previous approach. Finally, we conclude this paper in Section V.

\section{BACKGROUND}

\section{A. Latin Hypercube Sampling (LHS)}

Latin Hypercube Sampling (LHS) is a variant of QuasiMC method, which has been widely used to efficiently spread samples into the entire sampling space without any overlapping [17] as shown in Fig. 2.

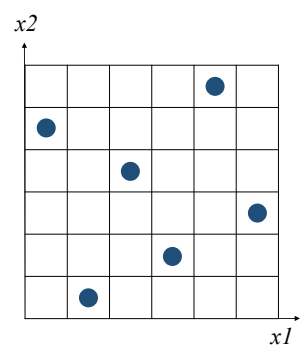

Fig. 2. An example of Latin hypercube sampling with 2 dimensions

In general, LHS method divides the cumulative distribution of each random variable into several intervals with equal probability and randomly chooses one sample from each interval. These samples can be transformed into the desired probabilistic distribution using the inverse cumulative function [17]. LHS is efficient because it guarantees that each small cell of the sampling space contains only one single sample.
In other words, any two Latin hypercube samples certainly occupy different positions and share no redundant information.

\section{B. Moment Matching for PDF Calculation}

Several approaches are proposed to determine the probabilistic distribution function (PDF) based on moments [13], [18]. In this work, we adopt the moment matching based approach to approximate the distribution of the circuit performance.

The probabilistic moments of a random variable and the time moments of a linear time-invariant (LTI) system are defined in (2) [19] and (1) [13] respectively.

$$
\begin{array}{r}
m_{y}^{k}=\int_{-\infty}^{+\infty} y^{k} \cdot p d f(y) \cdot d y \\
\widehat{m}_{t}^{k}=\frac{(-1)^{k}}{k !} \cdot \int_{-\infty}^{+\infty} y^{k} \cdot h(t) \cdot d t
\end{array}
$$

Alternatively, the time moments can also be represented as

$$
\widehat{m}_{t}^{k}=\sum_{r=1}^{M} \frac{a_{r}}{b_{r}^{k+1}}
$$

where $a_{r}$ and $b_{r}(r=1,2, \cdots, M)$ are the residues and poles of the LTI system, respectively. There is a known way to represent the impulse response $h(t)$ the LTI system as

$$
\begin{aligned}
h(t) & =\sum_{r=1}^{M} a_{r} \cdot e^{a_{r} \cdot t}, t>0 \\
& =0, t<0
\end{aligned}
$$

By matching the $m_{y}^{k}$ to $\widehat{m}_{t}^{k}$ and solving $a_{r}$ and $b_{r}$ in equation (3), we can derive the circuit performance distribution $p d f(y)$ of the given probabilistic moments $m_{y}^{k}$ as a closed-form expression in (4) [13].

\section{Aging Effects on Transistors}

As reported in previous works, the transistor parameter that has the most significant degradation is the threshold voltage $\left(V_{t h}\right)$. In order to simplify the degradation analysis, most of the previous works assume that only $V_{t h}$ is changed over time. The same assumption is also adopted in this work. The change of the threshold voltage of a-Si thin-film transistors (TFT) $(\triangle$ $\left.V_{t h}^{t_{s}}\right)$ with degradation at stress time $\left(t_{s}\right)$ can be expressed as (5) and (6) [20]. In these equations, the stress time $t_{s}$ is the time period after the initial time $\left(t_{0}\right)$, which is a usergiven value that represents the target lifetime. $\alpha$ and $\beta$ are two process-dependent parameters. According to the reported data in [20], this degradation model shows good consistency with real measurement results.

$$
\begin{array}{r}
\Delta V_{t h}^{t_{s}}=\left(V_{g s}-V_{t h}\right) \cdot\left\{1-\exp \left[-\left(\frac{t_{s}}{\tau}\right)^{\beta}\right]\right\} \\
\tau=k \cdot\left(V_{g s}-V_{t h}\right)^{\frac{1-\alpha}{\beta}}
\end{array}
$$




\section{BEHAVIOR MODELING CONSIDERING AGING EFFECTS}

\section{A. Problem Formulation and Algorithm Overview}

Considering the process variations, the circuit parameters, such as threshold voltage $\left(V_{t h}\right)$ and transistor width $(W)$, are modeled as random variables according to the models provided by the foundry. Those parameters form a random parameter vector $S=\left[X_{1}, X_{2}, \cdots, X_{N}\right]$. Stochastic behavior modeling analyzes the distribution of the circuit performance metric $Y$ by simulating a large number of circuits (a.k.a. samples) whose parameter vectors are $\left\{s_{1}, s_{2}, \cdots, s_{M}\right\}$ respectively.

In addition, the aging effect impacts these circuit parameters and introduces another dimension to the analysis. To understand the life cycle of a design, designers need to analyze the stochastic model of a full aging history, which requires to simulate $M$ samples at every aging time step, and leads to prohibitively long simulation time.

In this paper, we propose an incremental stochastic modeling approach, which reduces the simulation time in two folds. First, an incremental LHS sampling approach is proposed to reduce the number of samples that are required to be simulated when time step updates. Instead of updating and re-simulating all the samples, we only incrementally update a small portion of samples. Second, we model the distribution of performance $Y$ using moment matching in the parameter space to further reduce the number of updated samples at each time step.

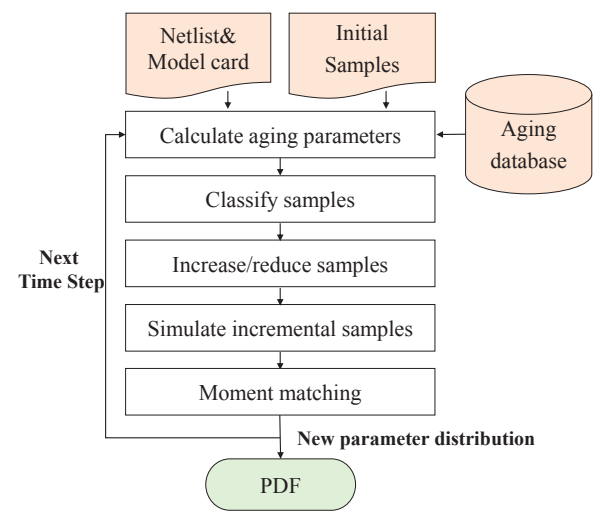

Fig. 3. Flowchart of the proposed incremental LHS for behavior modeling

The overall flow of the proposed algorithm is illustrated in Fig. 3. First, the information of circuit such as parameter distribution, circuit performance and PDF will be initialized at the first step. In the next step, new parameters after aging will be calculated to obtain the performance degradation. Then, Latin Hypercube Samples are obtained to generate the performance distribution for the initial PDF, and the new PDF in the next time step will be fitted based on the this PDF. In the next time step, the moments in the parameter space are adjusted by adding or removing some samples. Using this method, the property of LHS can be kept, and only partial samples need to be re-simulated.

\section{B. Incremental Latin Hypercube Sampling}

For each parameter, LHS divides the sample space into $M$ equally probable intervals and makes sure samples are evenly distributed in these intervals. Considering the aging effect, the distribution of a parameter may change over time. However, the distributions over the adjacent time steps will not change drastically because aging affects transistor parameters gradually. As an example, the original distribution of $V_{t h}$ on a fresh $8 \mu \mathrm{m}$ flexible TFT and the distribution after 100s aging of the same transistor are illustrated as $h^{0}$ and $h^{100}$ in Fig. 4. After 100 s aging, we can observe that the distribution is only slightly shifted to the right hand side, while there is a large portion of overlap between these two distributions.

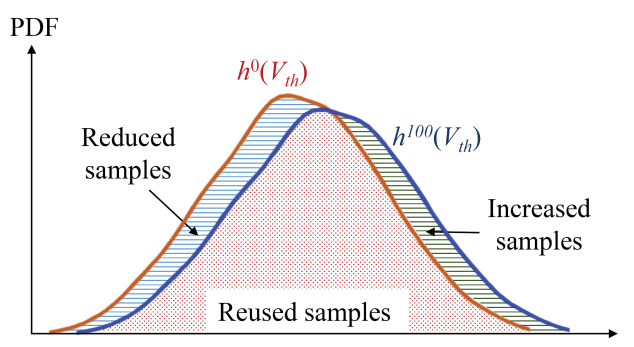

Fig. 4. Overlap of the original $V_{t h}$ distribution and that after aging

Based on the observation, we can obtain the new distribution in time $=100 \mathrm{~s}$ by adding a few new samples to those original samples and removing some redundant samples from them, while keeps the majority of samples. That is the motivation of the proposed incremental LHS sampling. More importantly, it is easy to keep the property of LHS in the incremental sampling, i.e. these samples are evenly distributed in each equally probable intervals in each dimension. This ensures the coverage in the sample space. In the following discussion, we denote the $V_{t h}$ as the $i^{t h}$ parameter, $X_{i}$, in the parameter vector $S$ without losing generality.

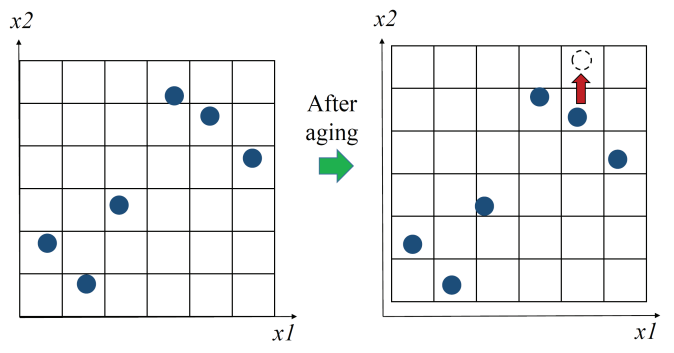

Fig. 5. Incremental Latin hypercube sampling with 2 dimensions

Take Fig. 5 as an example. Same to those $M$ initial LHS samples before aging, we divide $M$ equally probable intervals after one aging time step. Ideally, there is exactly one sample per interval. Because of the distribution shifting, however, an interval may cover multiple samples or no sample. In this case, the incremental LHS removes the "extra" samples in some intervals and adds them to empty intervals by incremental sampling, i.e. changing the $X_{i}$ value of the "extra" sample into a random value in the empty interval.

\section{Selecting Incremental Samples}

To determine the samples to be increased, reused and reduced, we use a greedy approach in this work. The pseudocode is elaborated in Algorithm 1. 


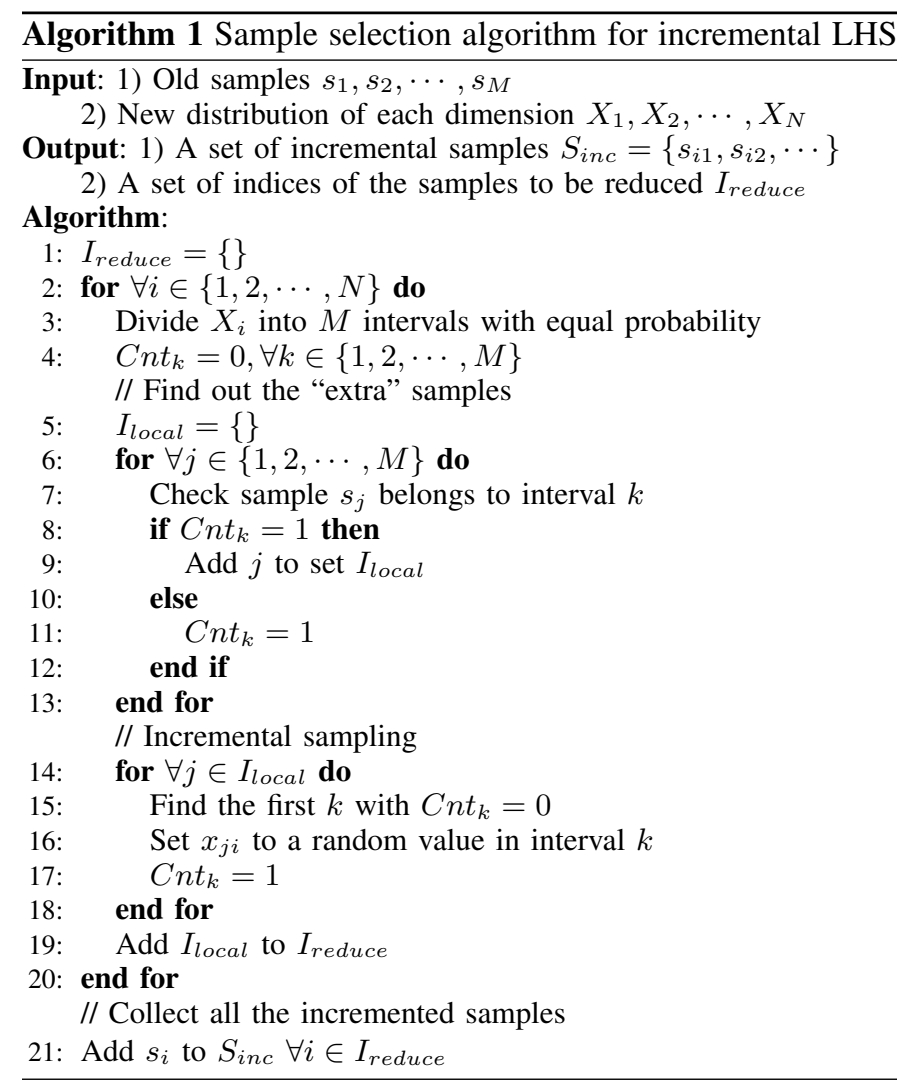

Assume there are $N$ parameters $S=\left[X_{1}, X_{2}, \cdots, X_{N}\right]$ with a total of $M$ samples $\left\{s_{1}, s_{2}, \cdots, s_{M}\right\}$, where the $i^{t h}$ sample $s_{i}$ is an $N$-dimension vector $\left[x_{i 1}, x_{i 2}, \cdots, x_{i N}\right]$. Algorithm 1 iterates all the $N$ dimensions and makes sure those $M$ samples are distributed in $M$ intervals with equal probability. In each dimension, the algorithm first locates the "extra" samples, and relocates them to the interval that does not have any samples in line 16 of Algorithm 1 . Since it only changes the value $x_{j i}$ in parameter $i$, it does not change the distribution on other parameters. Therefore, by iterating this process for each parameter, the new samples still satisfy the property of LHS by any means.

\section{Incremental Moment Matching}

For PDF analysis, equation (3) has to be evaluated to approximate the integral with several representative samples. In particular, the $k t h$-order probabilistic moment $m_{y}^{k}$ can be estimated as shown in (7), where $x_{j}$ are the $j$ th samples of $\widehat{x}$ using sampling method. Those samples can be classified into two types: reused samples $S_{\text {reused }}$ and incremental samples $\left(S_{\text {incr }}\right)$. The $S_{\text {reused }}$ has $\mathrm{N}$ samples, which includes sample set $j=1,2, \cdots, N$. Also, the $S_{i n c r}$ has M samples, which includes sample set $l=1,2, \cdots, M$ as shown in (7). $f\left(\overrightarrow{x_{j}}\right)$ is the performance merit of the circuit with input $\overrightarrow{x_{j}}$. This approach is actually the sampled form of the expectation value $\mathbb{E}\left(y^{k}\right)$ and only utilizes these representative samples $f\left(\overrightarrow{x_{j}}\right)$.

$$
\begin{aligned}
\widehat{m_{y}^{k}} & =\frac{(-1)^{k}}{k !} \cdot \int_{-\infty}^{+\infty} y^{k} \cdot p d f(y) d y=S_{\text {resued }}+S_{\text {incr }} \\
& \simeq\left\{\frac{1}{N} \sum_{j=1}^{N} f\left(\overrightarrow{x_{j}}\right)^{k}\right\}+\left\{\frac{1}{M} \sum_{l=1}^{M} f\left(\overrightarrow{x_{l}}\right)^{k}\right\}
\end{aligned}
$$

The next step is to re-analyze the residues $a_{r}$ and the poles $b_{r}$ in (3) with high-order moments so that the impulse response $h(t)$ in (4) can be evaluated to approximate $p d f(y)$. Consequently, (3) results in a nonlinear equation system as shown in (8).

$$
-\left[\begin{array}{c}
\frac{a_{1}}{b_{1}}+\frac{a_{2}}{b_{2}} \cdots \\
\frac{a_{1}}{b_{1}^{2}}+\frac{a_{2}}{b_{2}^{2}} \cdots \\
\vdots \\
\frac{a_{1}}{b_{1}^{(M+N)}}+\frac{a_{2}}{b_{2}^{(M+N)}} \cdots
\end{array}\right]=\left[\begin{array}{c}
\widehat{m}_{y}^{1} \\
\widehat{m}_{y}^{2} \\
\vdots \\
\widehat{m}_{y}^{(M+N)}
\end{array}\right]
$$

\section{EXPERIMENTAL RESULTS}

\section{A. Experiment Setup}

In this section, the proposed stochastic behavioral modeling for aging analysis is verified through several examples. Since flexible electronics have more severe aging than conventional CMOS, the proposed approach is evaluated on an OPA circuit [21] with ITRI $a-S i 8 \mu m$ technology as shown in Fig. 6. In addition, a 4-bit digital-to-analog convertor (DAC) using the same a-Si process for flexible electronics is implemented to verify the proposed approach. The DAC architecture is presented in Fig. 8. In the experiments, the $V_{d d}$ is configured as $25 \mathrm{~V}$ because flexible TFTs usually require high supply voltage to provide enough electron mobility.

In these two circuits, each transistor is modeled with four process variation parameters, channel width $(W)$, length $(L)$, threshold voltage, $\left(V_{t h}\right)$ and mobility $(\mu)$. In particular, flexible electronics are usually associated with large variations on $V_{t h}$ and $\mu$. According to previous work in [22], the relative standard deviation (std) of $V_{t h}$ and $\mu$ are modeled as $\pm 20 \%$ and $\pm 15 \%$ respectively, while the relative $s t d$ of device physical sizes ( $W$ and $L)$ are modeled as $\pm 3 \%$. The aging effect is calculated according to the model proposed in [20]. We analyze the aging effects with target lifetime of 100, 1000, and 10000 seconds, while the aging time step is configured as 100 seconds.

All the circuits are simulated using HSPICE, and the proposed algorithm is implemented in MATLAB. Instead of measuring the actually simulation time, we count the number of simulation runs to calculate the speedup. We also implement the following approaches for comparison purpose:

1) MC method: Calculate the probabilistic distributions from a large number of random samples. At each time step, all these samples need to be re-simulated with the updated transistor parameters. In particular, we implement two MC simulations, one with 6000 samples to serve as gold standard, while the 3000 -sample MC is more efficient but less accurate.

2) Quad Model for WCD: Quadratic degradation model is used to predict the parameter variations with aging effects as mentioned in [4].

3) Proposed method: Reliability and variability are considered simultaneously in the proposed stochastic behavioral modeling. We generate samples using incremental LHS to reduce the re-simulation cost, and moment matching in performance space is used to obtain the PDF efficiently. 


\section{B. Experimental Results of OPA circuit}

The differential OPA circuit is illustrated in Fig. 6, where the voltage gain is considered as the performance metric in this OPA circuit. In this particular technology, only n-type TFT is used for OPA circuit. We can also notice that a positive feedback is used to boost the voltage gain.

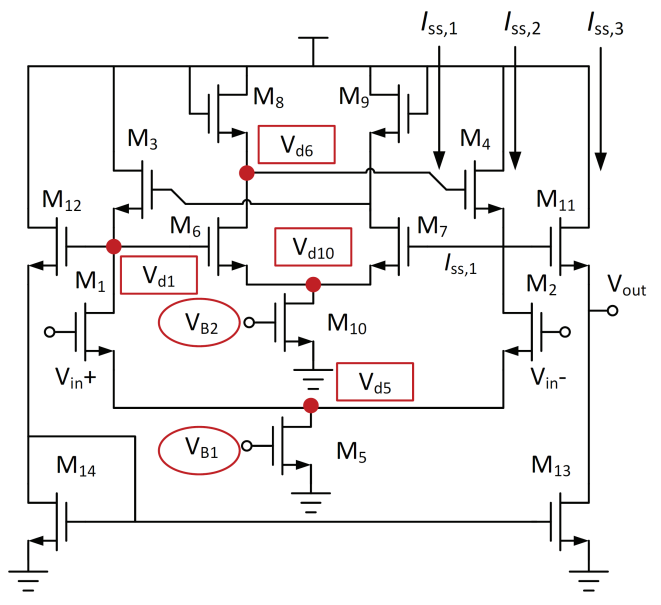

Fig. 6. The schematic of the operational amplifier [21]

1) Accuracy of the PDF Approximation: we evaluate the overall accuracy of the PDF compared with the gold standard, the MC simulation with 6000 samples. The PDF of this OPA circuit is plotted in Fig. 7 when the time=10000s. Here, the solid line is the PDF approximated by the proposed algorithm, which agrees to histogram of $6000 \mathrm{MC}$ samples. Compared with the gold standard, the proposed method achieves $99 \%$ accuracy with all time step configurations because the property of LHS can be kept at all time. With the quadratic model [4], the accuracy degrades quickly when the operation time becomes longer. The average accuracy of quadratic model is only $83 \%$ from time $=0$ to time $=10000 \mathrm{~s}$.

2) Efficiency Comparison: In the proposed approach, 500 samples is used to build the behavior model at the initial time step (time $=0 \mathrm{~s}$ ). Benefit from the incremental sampling, only a few samples are generated and simulated at each time step. Compared to the golden model, $243 \mathrm{x}$ average speedup can be obtained from $\mathrm{t}=0 \mathrm{~s}$ to $\mathrm{t}=10000 \mathrm{~s}$. The quadratic model requires 2000 samples to build the performance model, and the other 2000 samples are required to obtain the aging ratio. However, the accuracy of quadratic model is still lower than the proposed method because of the prediction error of the non-linear aging rate.

\section{Experimental Results of DAC circuit}

In the second experiment, a fairly complex 4-bit DAC circuit [21] is used to evaluate the proposed algorithm. It consists of a resistor string, a unity-gain buffer, and onchip switches. The resistor string uses two identical resistors for voltage division. Controlled by a 4-bit binary input, the switches are implemented by N-type TFTs for level selection. As shown in Fig. 8, an OPA is configured as a voltage follower to serve as the output buffer. In this circuit, the integral nonlinearity (INL) is considered as the performance metric, which is a term describing the maximum deviation between the ideal outputs of a DAC.
TABLE I. EXPERIMENTAL RESULTS COMPARISON OF OPA CIRCUIT

\begin{tabular}{|c|c|c|c|c|c|}
\hline Time $(s)$ & & MC (6k) & MC (3k) & Quad & Proposed \\
\hline \multirow{2}{*}{0} & Accuracy & $100 \%$ & $98 \%$ & $99 \%$ & $99 \%$ \\
& \# of Samples & 6000 & 3000 & $2000+2000$ & 500 \\
\hline \multirow{2}{*}{100} & Accuracy & $100 \%$ & $97 \%$ & $92 \%$ & $99 \%$ \\
& \# of Samples & 6000 & 3000 & - & 13 \\
\hline \multirow{2}{*}{1000} & Accuracy & $100 \%$ & $97 \%$ & $85 \%$ & $99 \%$ \\
& \# of Samples & 6000 & 3000 & - & 16 \\
\hline \multirow{2}{*}{10000} & Accuracy & $100 \%$ & $97 \%$ & $74 \%$ & $99 \%$ \\
& \# of Samples & 6000 & 3000 & - & 29 \\
\hline \multirow{3}{*}{ Overall } & Accuracy & $100 \%$ & $97 \%$ & $83 \%$ & $99 \%$ \\
& \# of Samples & 600000 & 300000 & 4000 & 2469 \\
\cline { 2 - 6 } & Speedup & $1 \mathrm{x}$ & $2 \mathrm{x}$ & $150 \mathrm{x}$ & $243 \mathrm{x}$ \\
\hline
\end{tabular}

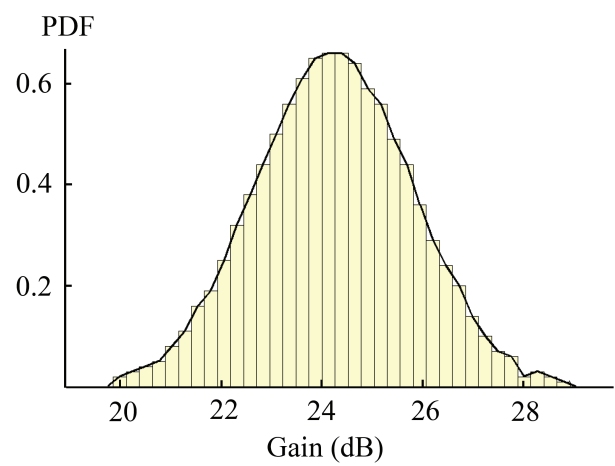

Fig. 7. The performance distribution comparison of OPA circuit at $\mathrm{t}=10000 \mathrm{~s}$

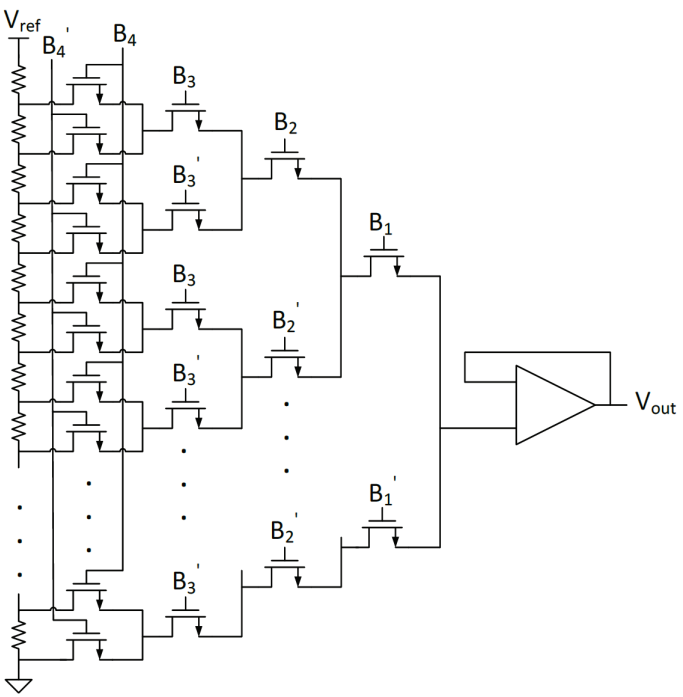

Fig. 8. The schematic of the digital-to-analog converter [21]

1) Accuracy of the PDF Approximation: With all time step configurations, the proposed method achieves $98 \%$ accuracy compared with the gold standard. Contrastingly, the quadratic model only achieves $82 \%$ accuracy on average. We can observe apparent decay on the accuracy if the quadratic model is used.

2) Efficiency Comparison: Same to the experiment on the OPA circuit, the proposed approach uses 500 samples to build up the behavior model at time $=0 \mathrm{~s}$. At the succeeding time steps, the required samples are greatly reduced but still keeping the same accuracy. The quadratic method requires 2000 samples to build the performance model, while another 2000 samples are required to obtain the aging ratio. However, 
the accuracy of quadratic model is still lower than the proposed method because of the prediction error of the non-linear aging rate. The number of simulated samples from $t=0 \mathrm{~s}$ to $\mathrm{t}=10000 \mathrm{~s}$ using different approaches are compared in Fig. 9.

TABLE II. EXPERIMENTAL RESULTS COMPARISON OF DAC CIRCUIT

\begin{tabular}{|c|c|c|c|c|c|}
\hline Time $(s)$ & & MC (6k) & MC (3k) & Quad & Proposed \\
\hline \multirow{2}{*}{0} & Accuracy & $100 \%$ & $98 \%$ & $97 \%$ & $99 \%$ \\
& \# of Samples & 6000 & 3000 & $2000+2000$ & 500 \\
\hline \multirow{2}{*}{100} & Accuracy & $100 \%$ & $97 \%$ & $89 \%$ & $98 \%$ \\
& \# of Samples & 6000 & 3000 & - & 12 \\
\hline \multirow{2}{*}{1000} & Accuracy & $100 \%$ & $96 \%$ & $82 \%$ & $98 \%$ \\
& \# of Samples & 6000 & 3000 & - & 19 \\
\hline \multirow{2}{*}{10000} & Accuracy & $100 \%$ & $96 \%$ & $73 \%$ & $98 \%$ \\
& \# of Samples & 6000 & 3000 & - & 33 \\
\hline \multirow{3}{*}{ Overall } & Accuracy & $100 \%$ & $97 \%$ & $82 \%$ & $98 \%$ \\
& \# of Samples & 600000 & 300000 & 4000 & 2478 \\
\cline { 2 - 6 } & Speedup & $1 \mathrm{x}$ & $2 \mathrm{x}$ & $150 \mathrm{x}$ & $242 \mathrm{x}$ \\
\hline
\end{tabular}

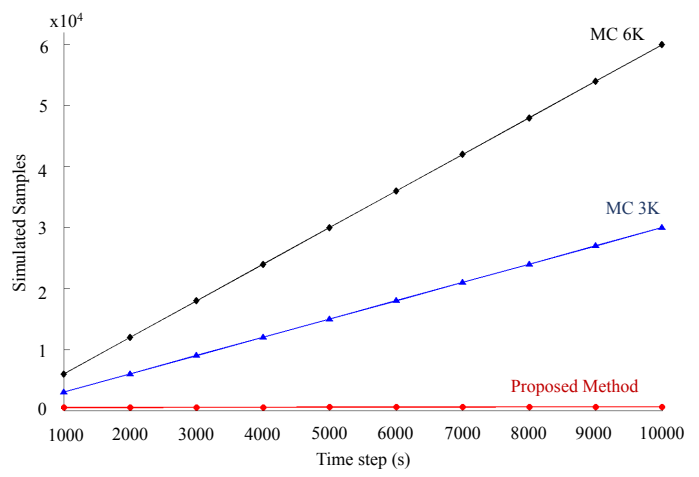

Fig. 9. The comparison results of simulated samples at each time step

\section{Conclusions}

In this paper, we propose an incremental Latin hypercube sampling approach to build the stochastic behavior models for analog/mixed-signal (AMS) circuits while simultaneously considering process variations and aging effects. By reusing previous sampling information, only a small portion of samples are incrementally updated to build an accurate stochastic model in different time steps, which significantly reduces the number of simulations for aging analysis. The accuracy of PDF estimation can be kept because of the property of LHS can be guaranteed in the proposed approach. Experiments on an operational amplifier and a 4-bit DAC circuits achieve $242 \mathrm{x}$ speedup over the monte carlo method with similar accuracy.

\section{REFERENCES}

[1] E. Maricau and G. Gielen, "Efficient variability-aware nbti and hot carrier circuit reliability analysis," Computer-Aided Design of Integrated Circuits and Systems, IEEE Transactions on, vol. 29, no. 12, pp. 18841893, Dec. 2010.

[2] D. Schroder and J. Babcock, "Negative bias temperature instability: Road to cross in deep submicron silicon semiconductor manufacturing," Journal of Applied Physics, vol. 94, no. 1, pp. 1-18, Jul. 2003.

[3] G. Gielen, E. Maricau, and P. De Wit, "Analog circuit reliability in sub32 nanometer cmos: Analysis and mitigation," in Design, Automation Test in Europe Conference Exhibition (DATE), 2011, Mar. 2011, pp. $1-6$.

[4] X. Pan and H. Graeb, "Reliability analysis of analog circuits using quadratic lifetime worst-case distance prediction," in Custom Integrated Circuits Conference (CICC), 2010 IEEE, Sep. 2010, pp. 1-4.
[5] W. Wu, Y. Shan, X. Chen, Y. Wang, and H. Yang, "FPGA accelerated parallel sparse matrix factorization for circuit simulations," in Reconfigurable Computing: Architectures, Tools and Applications. Springer, 2011, pp. 302-315.

[6] W. Wu, F. Gong, R. Krishnan, L. He, and H. Yu, "Exploiting parallelism by data dependency elimination: A case study of circuit simulation algorithms," Design Test, IEEE, vol. 30, no. 1, pp. 26-35, Feb 2013.

[7] X. Chen, W. Wu, Y. Wang, H. Yu, and H. Yang, "An ESchedulerBased data dependence analysis and task scheduling for parallel circuit simulation," IEEE Trans. Circuits Syst. II, Exp. Briefs, vol. 58, no. 10, pp. $702-706$, oct. 2011.

[8] X. Pan and H. Graeb, "Degradation-aware analog design flow for lifetime yield analysis and optimization," in Electronics, Circuits, and Systems, 2009. ICECS 2009. 16th IEEE International Conference on, Dec. 2009, pp. 667-670.

[9] M. Arulampalam, S. Maskell, N. Gordon, and T. Clapp, "A tutorial on particle filters for online nonlinear/non-gaussian bayesian tracking," Signal Processing, IEEE Transactions on, vol. 50, no. 2, pp. 174-188, Feb. 2002.

[10] A. Singhee and R. Rutenbar, "From finance to flip flops: A study of fast quasi-monte carlo methods from computational finance applied to statistical circuit analysis," in Quality Electronic Design, 2007. ISQED '07. 8th International Symposium on, Mar. 2007, pp. 685-692.

[11] J. Jaffari and M. Anis, "On efficient lhs-based yield analysis of analog circuits," Computer-Aided Design of Integrated Circuits and Systems, IEEE Transactions on, vol. 30, no. 1, pp. 159-163, Jan. 2011.

[12] S. Vrudhula, J. Wang, and P. Ghanta, "Hermite polynomial based interconnect analysis in the presence of process variations," ComputerAided Design of Integrated Circuits and Systems, IEEE Transactions on, vol. 25, no. 10, pp. 2001-2011, Oct. 2006.

[13] F. Gong, S. Basir-Kazeruni, L. He, and H. Yu, "Stochastic behavioral modeling and analysis for analog/mixed-signal circuits," ComputerAided Design of Integrated Circuits and Systems, IEEE Transactions on, vol. 32, no. 1, pp. 24-33, Jan. 2013.

[14] X. Li, J. Le, P. Gopalakrishnan, and L. Pileggi, "Asymptotic probability extraction for nonnormal performance distributions," Computer-Aided Design of Integrated Circuits and Systems, IEEE Transactions on, vol. 26, no. 1, pp. 16-37, Jan. 2007.

[15] R. Krishnan, W. Wu, F. Gong, and L. He, "Stochastic behavioral modeling of analog/mixed-signal circuits by maximizing entropy," in Quality Electronic Design (ISQED), 2013 14th International Symposium on, Mar. 2013, pp. 572-579.

[16] H. Kumamoto, T. Tanaka, and K. Inoue, "A new monte carlo method for evaluating system-failure probability," Reliability, IEEE Transactions on, vol. R-36, no. 1, pp. 63-69, Apr. 1987.

[17] M. McKay, R. Beckman, and W. Conover, "A comparison of three methods for selecting values of input variables in the analysis of output from a computer code," Technometrics, pp. 239-245, 1979.

[18] C. Fang, F. Yang, X. Zeng, and X. Li, "Bmf-bd: Bayesian model fusion on bernoulli distribution for efficient yield estimation of integrated circuits," in Design Automation Conference (DAC), 2014 51st ACM/EDAC/IEEE, Jun. 2014, pp. 1-6.

[19] X. Li, J. Le, P. Gopalakrishnan, and L. Pileggi, "Asymptotic probability extraction for non-normal distributions of circuit performance," in Computer Aided Design, 2004. ICCAD-2004. IEEE/ACM International Conference on, Nov. 2004, pp. 2-9.

[20] S.-E. Liu, C.-P. Kung, and J. Hou, "Estimate threshold voltage shift in a-si:h tfts under increasing bias stress," Electron Devices, IEEE Transactions on, vol. 56, no. 1, pp. 65-69, Jan. 2009.

[21] Y.-C. Tarn, P.-C. Ku, H.-H. Hsieh, and L.-H. Lu, "An amorphous-silicon operational amplifier and its application to a 4-bit digital-to-analog converter," Solid-State Circuits, IEEE Journal of, vol. 45, no. 5, pp. 1028-1035, May 2010.

[22] E.-H. Ma, W.-E. Wei, H.-Y. Li, J.-M. Li, I.-C. Cheng, and Y.-H. Yeh, "Flexible tft circuit analyzer considering process variation, aging, and bending effects," Display Technology, Journal of, vol. 10, no. 1, pp. 19-27, Jan. 2014. 